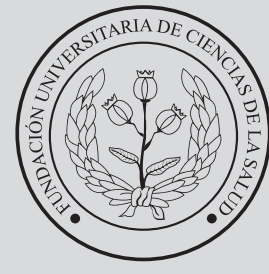

FUCS
Re 1

\title{
Criptococosis diseminada en paciente positivo para COVID-19
}

Héctor Fabio Restrepo MD Cristian Gutiérrez Lara MD $^{\mathbf{b}}$ Mauricio Milanés MDc

\section{Disseminated cryptococcosis in a patient with COVID-19}

${ }^{a}$ Médico Veterinario Zootecnista, Médico Cirujano, Especialista en Epidemiología. MSc Epidemiología. PhD (C), Gestión de Proyectos. Docente Investigador Facultad de Medicina, Fundación Universitaria de Ciencias de la Salud, Bogotá DC, Colombia.

${ }^{b}$ Médico Cirujano, Universidad El Bosque, Bogotá DC, Colombia.

' Médico Cirujano, Especialista en Medicina Familiar, Especialista en Medicina Crítica y Cuidado Intensivo. Bogotá DC, Colombia.

\section{R E S U M EN}

Paciente de 59 años quien ingresa al servicio de urgencias con un cuadro de 2 días de evolución de cefalea holocraneana de intensidad moderada 7/10, picos febriles no cuantificados, disnea grado III, adinamia, hiporexia, ageusia y tos no productiva, refiriendo contacto estrecho con hermana confirmada para infección por COVID-19, por lo que se investiga antígeno para COVID-19 con resultado positivo. Se confirma la existencia del síndrome respiratorio agudo grave (SARS-CoV-2) con coinfección por Cryptococus neoformans. El manejo con corticoide sistémico genera un importante inmunocompromiso que predispone al paciente a coinfecciones por gérmenes comunes y oportunistas, como lo es la infección por Cryptococcus neoformans/ Gatti. En este caso la infección por COVID-19 y el desarrollo de SARS-CoV-2 fue la etiología de la infección micótica por cryptococo.

Palabras clave: criptococosis, COVID-19, cefalea holocraneana.

(C) 2021 Fundación Universitaria de Ciencias de la Salud - FUCS Este es un artículo Open Access bajo la licencia CC BY-NC-ND (http://creativecommons.org/licenses/by-nc-nd/4.0/).

Historia del artículo:

Fecha recibido: mayo 12 de 2021 Fecha aceptado: junio 28 de 2021

\section{INFORMACIÓN DEL ARTÍCULO}

Autor para correspondencia.

Dr. Héctor Fabio Restrepo

hfrestrepo@fucsalud.edu.co
DOI

10.31260/RepertMedCir.01217372.1209 
Here, we report a case of a 59-year-old patient with a 2-day history of moderate intensity (7/10) holocranial headache, unquantified febrile peaks, grade III dyspnea, adynamia, hyporexia, ageusia and nonproductive cough admitted to the emergency department. He referred close contact to a sister with confirmed COVID-19. His COVID-19 antigen test results were positive. Severe acute respiratory syndrome (SARS-CoV-2) associated with Cryptococcus neoformans infection was confirmed. The use of systemic corticosteroid therapy leads to immunocompromise and predisposes the patient to common and opportunistic infections such as Cryptococcus neoformans and Cryptococcus gatiii infection. In this case COVID-19 infection and the development of SARS-CoV-2 was the etiology of the Cryptococcus fungal infection.

Key words: cryptococcosis, COVID-19, holocranial headache.

(C) 2021 Fundación Universitaria de Ciencias de la Salud - FUCS. This is an open access article under the CC BY-NC-ND license (http://creativecommons.org/licenses/by-nc-nd/4.0/).

\section{INTRODUCCIÓN}

La enfermedad por coronavirus de 2019 COVID-19 es una patología ocasionada por el nuevo coronavirus del síndrome respiratorio agudo grave (SARS-CoV-2), ${ }^{1}$ teniendo un gran impacto sociocultural y económico a nivel mundial, reportándose más de 100 millones de casos confirmados y cerca de 2.4 millones de muertes. En enero 2020 se publicó un estudio realizado en Wuhan, China, en donde se describen las principales características, evidenciando que $49 \%$ de los pacientes se encontraban entre 25 y 49 años, $34 \%$ estaba entre 50 y 64 años, con un promedio de edad de 49 años. Cerca de la mitad presentaban alguna comorbilidad previa, reportándose diabetes 20\%, hipertensión 15\% y enfermedad cardiovascular 15\%; $98 \%$ presentaba fiebre como principal sintomatología, seguida de tos $76 \%$ y disnea 55\%. El hallazgo principal al ingreso hospitalario fue leucopenia y linfopenia en $25 \%$ y $63 \%$ respectivamente, asociado con marcadores de severidad, como dímero D, troponina, ferritina y deshidrogenasa láctica. ${ }^{2}$ Estos hallazgos suponen impacto directo de la infección viral sobre el sistema inmune favoreciendo la presencia exagerada de citosinas, gran liberación y consumo de interleucina 1 (IL-1) e interleucina 6 (IL-6), por lo que se denominó a este fenómeno "tormenta de citocinas" comparable con la linfohistiocitosis (sHLH, por sus siglas en inglés) descrita en los pacientes con infección por el coronavirus MERS-CoV. ${ }^{3}$

Los pacientes con esta entidad pueden permanecer por tiempo prolongado en la unidad de cuidado intensivo (UCI) y $80 \%$ de ellos llegan a requerir ventilación mecánica invasiva. ${ }^{4,5}$ Los críticamente enfermos por COVID-19 se asocian con un elevado riesgo de presentar neumonía nosocomial, en especial cuando requieren ventilación mecánica invasiva, con aumento del riesgo de neumonía asociada con el ventilador en comparación con pacientes sin infección por COVID-19. ${ }^{6}$ Los resultados preliminares del estudio RECOVERY mostraron que en pacientes hospitalizados con COVID-19 que requirieron ventilación mecánica invasiva el uso de dexametasona durante 10 días disminuyó la mortalidad a 28 días en $12.3 \%$, mientras que aquellos hospitalizados que solo recibieron oxígeno sin soporte ventilatorio se redujo la mortalidad en $4.1 \%{ }^{7} \mathrm{Se}$ ha encontrado con frecuencia compromiso hematológico y alteraciones de coagulabilidad, que impactan de manera directa el funcionamiento multisistémico con exagerada lesión endotelial, generando microtrombos a nivel microvascular, causando disfunción multiorgánica y aumento en el score de SOFA. En contexto de infección por COVID-19 el articulo de Yang y col. reportó una sensibilidad de $85.2 \%$ y especificidad de $80.4 \%$ cuando se tomó un punto de corte de 2, mientras que cuando se realizó $\geq 5$ puntos, la sensibilidad fue de $100 \%$ y especificidad $95.4 \%$. Esto sugiere que aquellos pacientes con un SOFA score $\geq 5$ tienen un riesgo de mortalidad mayor en comparación con aquellos con score $<5 .{ }^{8}$ Además se ha encontrado un mayor riesgo de infecciones micóticas en los pacientes que sufren SARS-CoV-2, evidenciando un aumento de la aspergilosis pulmonar invasiva (IPA, por sus siglas en inglés) como una complicación importante en dichos pacientes. ${ }^{9,10}$

La criptococosis, ocasionada por las especies del complejo Cryptococcus neoformans ( $C$. neoformans/C. gattii), afecta sobre todo a los inmunocomprometidos. En la población colombiana se presenta una incidencia anual de 2.4 casos por millón de habitantes a pesar de que principalmente se asocia con infección por VIH y en 13\% de los casos no se identifica un factor predisponente. La infección del sistema nervioso central (SNC) es la forma de presentación más frecuente y grave, manifestándose como una meningitis subaguda o crónica, caracterizada por cefalea, náuseas, vómito, fiebre y alteración del estado de conciencia. En menor medida 
se encuentran el compromiso pulmonar, afectación de piel y ganglios $\mathrm{u}$ otro órgano. Para su diagnóstico el líquido cefalorraquídeo es la muestra de elección, reportando una presión de apertura aumentada al momento de la punción lumbar, asociada con leucocitos aumentados de predominio linfocitario, hiperproteinorraquia e hipoglucorraquia, con blastoconidias encapsuladas redondeadas u ovaladas de 4-6 $\mu \mathrm{m}$ de diámetro, evidenciadas con la técnica de exclusión de tinta china. El esquema de tratamiento depende del órgano afectado y su presentación clínica, considerándose la forma más severa la diseminada o con compromiso del SNC. En casos graves de compromiso pulmonar se sugiere realizar tratamiento con el esquema propuesto para infección diseminada o con compromiso del SNC. Ante la presencia de criptococomas cerebrales se debe considerar el tratamiento quirúrgico ante tumoraciones $\geq 3 \mathrm{~cm}$ de diámetro con marcado efecto de masa y en áreas accesibles del encéfalo. Cuando hay incertidumbre diagnóstica o falla terapéutica, el tratamiento alternativo consta de itraconazol, voriconazol (200 mg/cada 12 horas VO) o posaconazol (400 mg/cada 12 horas VO) durante 6 a 12 meses. ${ }^{11}$

\section{PRESENTACIÓN DEL CASO}

Paciente de 59 años que ingresa al servicio de urgencias con cuadro de 2 días de evolución de cefalea holocraneana de intensidad moderada 7/10, picos febriles no cuantificados, disnea grado III, adinamia, hiporexia, ageusia y tos no productiva, refiriendo contacto estrecho con hermana confirmada para infección por COVID-19, por lo que se realiza antígeno para COVID-19 con resultado positivo. Paciente sin requerimiento de $\mathrm{O} 2$ suplementario, sin signos de dificultad respiratoria, se da egreso hospitalario sin antibioticoterapia, manejo con antipirético oral (acetaminofén) y signos de alarma.

\section{Antecedentes}

Patológicos: evento cerebrovascular isquémico hace 10 años, accidente isquémico transitorio en 2 ocasiones, hipertensión, obesidad, dislipidemia, SAHOS y artritis gotosa. Farmacológicos: enalapril $20 \mathrm{mg} / 12 \mathrm{~h}$, metoprolol $50 \mathrm{mg} /$ día, atorvastatina $40 \mathrm{mg} /$ día, clopidogrel $75 \mathrm{mg} /$ día, amlodipino $10 \mathrm{mg} /$ día, lansoprazol $30 \mathrm{mg} /$ día, alopurinol $100 \mathrm{mg} /$ día. Tóxicos/exposicionales: ex-fumador hace 20 años.

\section{Evolución}

Cuatro días después reingresa con empeoramiento de la sintomatología, presentando disnea en reposo con desaturación hasta $60 \%$, requiriendo $\mathrm{O} 2$ suplementario por cánula nasal (Fio2 28\%) indicando manejo en unidad de cuidados intermedios, con inicio de cubrimiento antibiótico con ampicilina/sulbactam y claritromicina, además de dexametasona a dosis de $6 \mathrm{mg} /$ día. Presenta deterioro clínico y patrón respiratorio con aumento de Fio2 hasta $90 \%$ por máscara de no reinhalación $15 \mathrm{~L} / \mathrm{min}$, reportando PaFi 54, indicando manejo en UCI, decidiendo intubación orotraqueal. Se calculan escalas de severidad APACHE II: 16 puntos; SOFA: 5 puntos NEWS 2: 7 puntos; COVID GRAM: 229 puntos.

Las imágenes al ingreso del paciente en la radiografía de tórax mostraron múltiples infiltrados alveolares e intersticiales con opacidades de distribución multilobar bilateral. La tomografía de tórax de alta resolución evidenció abundantes opacidades en configuración de vidrio esmerilado a nivel multilobar, sumadas a procesos consolidativos del espacio aéreo (figura 1).

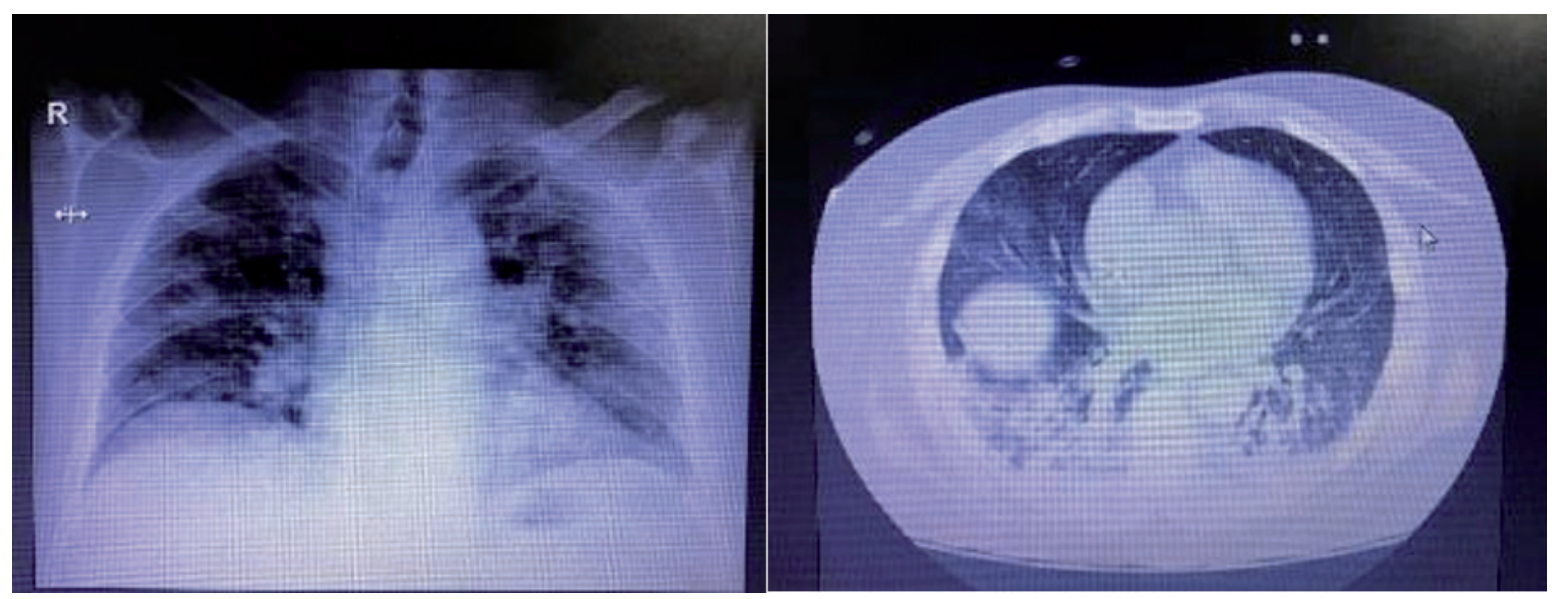

Figura 1. Radiografía y tomografía de ingreso. Fuente: los autores. 
Se inician maniobras de pronación y relajación neuromuscular con cisatracurio durante 48 horas presentando respuesta favorable, por lo que se completaron 5 ciclos de pronación hasta conseguir PaFi $>150$. Aparece con leucocitosis y neutrofilia en ascenso por lo cual se suspende ampicilina sulbactam y claritromicina y se inicia manejo con meropenem $2 \mathrm{~g}$ cada 8 horas y clindamicina $600 \mathrm{mg}$ cada 6 horas, posterior a la toma de hemocultivos. Por evolución ventilatoria favorable se realizó extubación sin complicaciones. Ante persistencia de leucocitosis y neutrofilia tras 48 horas de escalonamiento antibiótico, se decidió suspender claritromicina. Por el alto riesgo de coinfección micótica se inició cubrimiento con fluconazol, tras este hallazgo se adicionó anfotericina B $0.9 \mathrm{mg} / \mathrm{k} /$ día y fluconazol $400 \mathrm{mg}$ cada 12 horas por parte del servicio de cuidado crítico y se solicitaron paraclínicos para descartar inmunosupresión por causas infecciosas, con resultado negativo.

Se observó infarto antiguo en ganglio basal izquierdo con gliosis y pérdida de volumen con marcada tortuosidad y dilatación de la arteria carótida interna y de la porción $\mathrm{ml}$ de la arteria cerebral media, sin hallazgos que sugirieran evento isquémico agudo (figura 2).

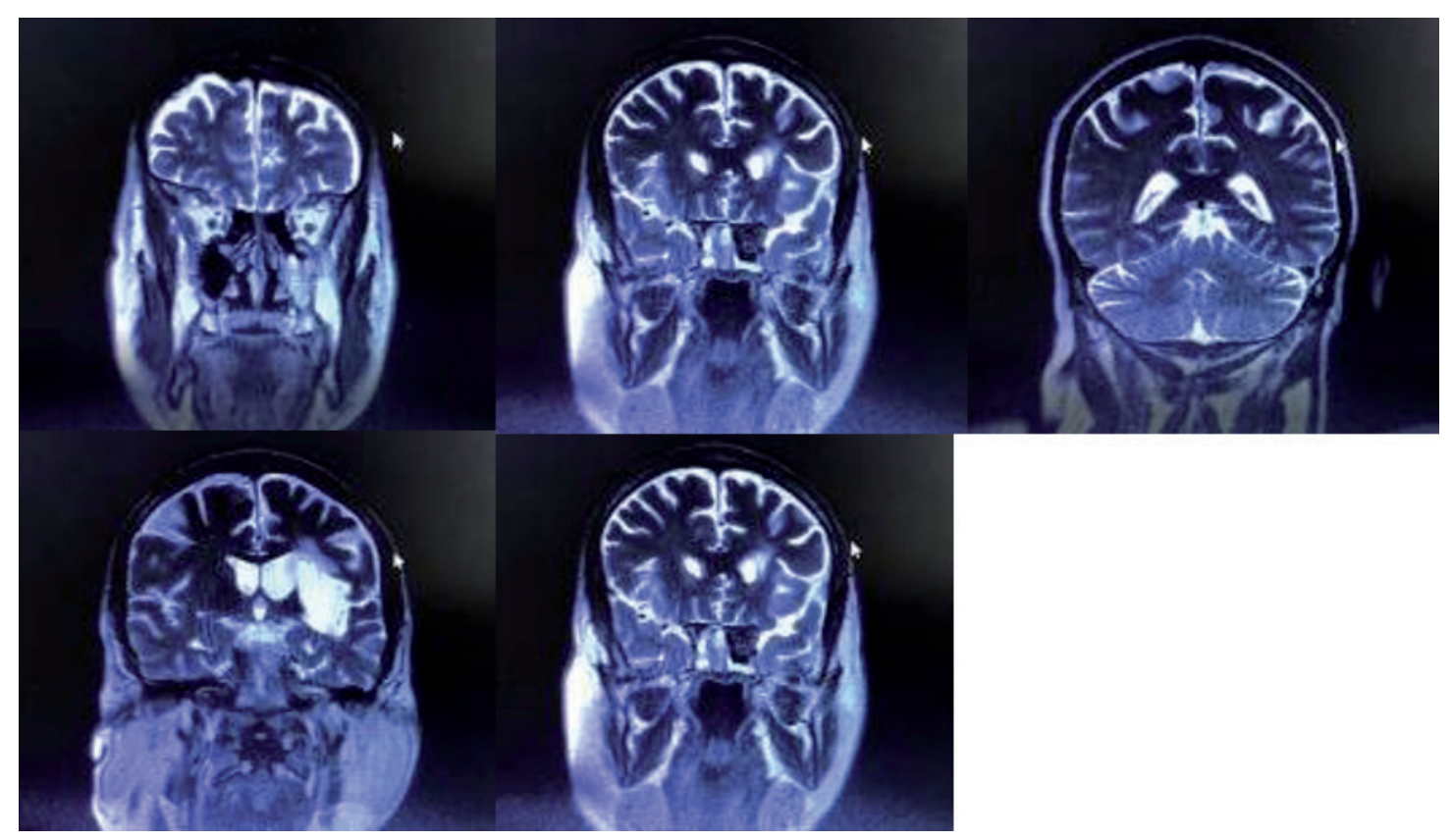

Figura 2. Imágenes diagnósticas. Fuente: los autores.

Presenta recuperación clínica progresiva tras completar intrahospitalariamente esquema de inducción y consolidación sin complicaciones, reportando hemocultivos negativos tomados a los 10 días del manejo antimicótico, con recuperación del estado de conciencia y sin déficit neurológico. Finalmente, se indica egreso hospitalario y manejo en casa con fluconazol en dosis de $200 \mathrm{mg}$ cada día para la fase de mantenimiento bajo seguimiento ambulatorio.

\section{DISCUSIÓN}

El actual reporte es un caso sin precedente de un paciente con diagnóstico de COVID-19 en quien se instaura síndrome respiratorio agudo grave (SARS-CoV-2) con coinfección por
Cryptococus neoformans. Al paciente se le calculó la escala SOFA al ingreso a la UCI con reporte de 5, sugiriendo un riesgo de mortalidad mayor de 33\%. Durante su estancia en cuidados intensivos presentó evolución satisfactoria del patrón respiratorio, con recuperación progresiva de los índices de oxigenación, respondiendo a las maniobras de pronación por 5 ciclos y relajación neuromuscular durante las primeras 48 horas posteriores al diagnóstico de SDRA, logrando así su extubación satisfactoria. A pesar de esto, ante la persistencia de signos de respuesta inflamatoria sistémica (SIRS) dado por leucocitosis con neutrofilia y taquicardia refleja, se decidió escalonamiento antibiótico y toma de hemocultivos, reportando a las 72 horas aislamiento de Cryptococus neoformans y Serratia marscecens en sangre. Tras este hallazgo se inició manejo con anfotericina B más fluconazol y se continuó cubrimiento antibiótico con 
carbapenémico. Tres días después el servicio de neurología realizó punción lumbar diagnóstica, la cual se reporta dentro de parámetros de normalidad.

En la enfermedad por COVID-19 se ha evidenciado un aumento de infección por otros gérmenes poco frecuentes en pacientes inmunocompetentes como es la causada por Aspergillus. En un estudio retrospectivo Koehler P. y col. describen el hallazgo de aspergilosis pulmonar invasiva (IPA por sus siglas en inglés) en 5 pacientes previamente inmunocompetentes ${ }^{10}$, mientras que Alanio A. y col. reportan diagnóstico de posible y probable IPA en 30\% y $4 \%$ respectivamente, de un total de 27 pacientes evaluados que requirieron estancia en UCI y ventilación mecánica invasiva secundaria a infección por COVID-19, sin antecedente de inmunosupresión. ${ }^{12}$

Cabe resaltar que al momento del diagnóstico de criptococosis, el paciente ya había completado el esquema con corticoide sistémico-dexametasona en dosis de $6 \mathrm{mg} /$ día por 10 días, lo cual agregado a la infección viral por COVID-19 pudo haberse asociado a un impacto sobre la respuesta inmunitaria hacia gérmenes nosocomiales $y$ oportunistas.

En cuanto al aspecto neurológico, se presentó persistencia de delirium hipoactivo tras el egreso de la UCI, considerándose mixto de etiología multifactorial, tanto por estancia prolongada en UCI como infecciosa, presentando recuperación progresiva sin secuelas.

En este caso llamó la atención el antecedente de un evento cerebrovascular isquémico hace 10 años y accidente isquémico transitorio en 2 ocasiones con ultima hospitalización hace 6 meses, razón por la cual se realizó perfil de autoinmunidad en hospitalización previa, con resultados de negatividad, descartando enfermedad autoinmune. Así mismo, el perfil infeccioso se reporta sin alteración, descartando inmunosupresión por VIH, hepatitis B, hepatitis C o Treponema pallidum. Tras reinterrogar al paciente y a los familiares, refieren antecedentes exposicionales a cacatúas y canarios australianos, siendo valorado por el servicio de neumología quienes consideraron que cursaba con criptococosis diseminada en paciente inmunocompetente. Es posible que este antecedente esté relacionado con exposición previa al criptococo y que la inmunosupresión indicada en el contexto de infección grave por SARS-CoV-2 haya generado la diseminación hematógena.

Consideramos que la infección por COVID-19 y el desarrollo de SARS-CoV-2 fue la etiología de la infección micótica por Cryptococus, teniendo en cuenta el gran impacto multisistémico y la disfunción multiorgánica instaurada por el agente viral como la principal causa de inmunosupresión predisponente a infecciones por este germen. Aunado a esto, el manejo desde el ingreso a unidad de cuidados intermedios con glucocorticoides (dexametasona) pudo favorecer al compromiso inmunológico ya instaurado por la enfermedad viral.

\section{REFEREN CI AS}

1. Accini Mendoza JL, Nieto Estrada VH, Beltrán López N, Ramos Bolaños E. Declaración de consenso en medicina crítica para la atención multidisciplinaria del paciente con sospecha o confirmación diagnóstica de COVID-19. Colombia: Asociación Colombiana de Medicina Crítica y Cuidado Intensivo AMCI; 2020. p. 7.

2. Huang C, Wang Y, Li X, Ren L, Zhao J, Hu Y, et al. Clinical features of patients infected with 2019 novel coronavirus in Wuhan, China. Lancet. 2020;395(10223):497-506. doi: 10.1016/S01406736(20)30183-5

3. Moore JB, June $\mathrm{CH}$. Cytokine release syndrome in severe COVID-19. Science. 2020;368(6490):473-4. doi: 10.1126/science. abb8925

4. Goyal P. Clinical Characteristics of Covid-19 in New York City. N Engl J Med 2020; 382:2372-2374. doi: 10.1056/NEJMc2010419

5. Grasselli G, Zangrillo A, Zanella A, Antonelli M, Cabrini L, Castelli A, et al. Baseline Characteristics and Outcomes of 1591 Patients Infected with SARS-CoV-2 Admitted to ICUs of the Lombardy Region, Italy. JAMA. 2020;323(16):1574-1581. doi: 10.1001/ jama.2020.5394

6. Maes M, Higginson E, Pereira-Dias J, Curran MD, Parmar S, Khokhar F, et al. Ventilator-associated pneumonia in critically ill patients with COVID-19. Crit Care. Crit Care. 2021 Jan 11;25(1):25. doi: 10.1186/s13054-021-03460-5

7. RECOVERY Collaborative Group, Horby P, Shen Lim W, Emberson JR, Mafham M, Bell JL, et al. Dexamethasone in Hospitalized Patients with Covid-19. N Engl J Med. 2021;384(8):693-704. doi: 10.1056/NEJMoa2021436

8. Yang Z, Hospital JC, Hu Q, Hospital JC, Huang F, Hosiptal JC, et al. The Prognostic Value of the SOFA Score in Patients with COVID-19 : A Retrospective, Observational Study. Res Sq. 2020. doi: 10.21203/rs.3.rs-67639/v1

9. Mercier T, Dunbar A, Veldhuizen V, Holtappels M, Schauwvlieghe A, Maertens J, et al. Point of care aspergillus testing in intensive care patients. Crit Care. 2020;24:642. doi: 10.1186/s13054-02003367-7

10. Koehler P, Cornely OA, Böttiger BW, Dusse F, Eichenauer DA, Fuchs F, et al. COVID-19 associated pulmonary aspergillosis. Mycoses. 2020;63(6):528-34. doi: 10.1111/myc.13096

11. Castañeda E, Lizarazo J. Protocolo de estudio y manejo de los pacientes con criptococosis A protocol for the study and management of patients with cryptococcosis Infectio Asociación Colombiana de Infectología. Infectio. 2012;16(3):123-5. doi: 10.1016/S0123-9392(12)70038-3

12. Alanio A, Dellière $S$, Fodil $S$, Bretagne $S$, Mégarbane B. Prevalence of putative invasive pulmonary aspergillosis in critically ill patients with COVID-19. Lancet Respir Med. 2020;8(6):e48-9. doi: 10.1016/S2213-2600(20)30237-X 